\title{
Subband energy in two-band $\delta$-doped semiconductors
}

\author{
F. Domínguez-Adame \\ Departamento de Física de Materiales, Universidad Complutense, 28040 Madrid, Spain
}

\begin{abstract}
We study electron dynamics in a two-band $\delta$-doped semiconductor within the envelope-function approximation. Using a simple parametrization of the confining potential arising from the ionized donors in the $\delta$-doping layer, we are able to find exact solutions of the Dirac-type equation describing the coupling of host bands. As an application we then consider Si $\delta$-doped GaAs. In particular we find that the ground subband energy scales as a power law of the Si concentration per unit area in a wide range of doping levels. In addition, the coupling of host bands leads to a depression of the subband energy due to nonparabolicity effects.
\end{abstract}

PACS numbers: 73.20.Dx; 85.42.+m; 71.25.Cx; 73.61.Ey

Typeset using REVTEX 


\section{INTRODUCTION}

Recently attention has been paid to planar- or $\delta$-doped semiconducting materials [1] (see Ref. [2] for a recent review) due to their potential application in ultra-high speed electronic and opto-electronic devices. Such doping profiles are generated by interrupting the crystal growth of the host material and evaporating the doping impurity during molecular-beam epitaxy. Under appropriate growth conditions, excellent confinement of dopant atoms has been achieved in GaAs:Si [3], Si:Sb [4], and InP:S [5]. As a consequence, the doping profile along the growth direction $z$ can be represented as $N(z)=N_{D} \delta(z)$, where $N_{D}$ is the twodimensional donor concentration. The above profile neglects the random distribution of donors in the $\delta$-doping layer, which is valid in the high density limit [6].

The analysis of the resulting electronic structure relies on the envelope function approximation [7]. Assuming parabolic bands, the envelope functions can be calculated by solving the Schrödinger-type equation for the one-electron potential, corresponding to decoupled host bands. This approach works fine in wide-gap semiconductors, provided that conductionand valence-band modulations are small. However a more realistic band-structure is required in narrow-gap semiconductors or in those devices whose band modulation is comparable to the magnitude of the gap, mainly due to nonparabolicity effects. In $\delta$-doped semiconductors large band modulation can be attained since it is possible to reach very high doping levels, typically larger than $10^{13} \mathrm{~cm}^{-2}$, thus leading to quantum confined states deep in the gap. This situation is even more dramatic in sawtooth superlattices, which consist of periodic alternating $n$ - and $p$-type $\delta$-doped sheets, separated by undoped material [8]. It is known that two-band models may successfully describe those nonparabolicity effects. The aim of this paper is to obtain exact solutions of two-band Hamiltonians for single $\delta$-doped layers, without requiring numerical techniques. As an illustration of our treatment, we will consider Si $\delta$-doped GaAs and compare the results with those obtained using simpler approaches. 


\section{MODEL}

For the calculation of the electron dynamics in $\delta$-doped semiconductors one requires the one-electron potential due to ionized donors. In the high concentration limit, several methods has been used, like the Thomas-Fermi semiclassical approach [9-11] the local density approximation (LDA) [12], and the Hartree method [13,14. In these methods, however, the computation of the one-electron potential relies on numerical techniques, so an analytical solution for the envelope functions and subband energies is no longer possible. However, Gold $a l$. 15 have proposed a closed form of the one-electron potential which brings accurate results in a wide range of doping levels. Hereafter we focus on GaAs, although we should stress that the treatment is completely general. In the high concentration limit, which we are interested in, the solution of the Poisson equation, assuming that the donor concentration presents a $\delta$-function profile, gives [15]

$$
V(z)=-g \exp \left(-\frac{|z|}{\alpha}\right),
$$

where $\alpha=38.0\left(N_{D} a^{* 2}\right)^{-1 / 3} \AA, g=38.1\left(N_{D} a^{* 2}\right)^{2 / 3} \mathrm{meV}$, with the effective Bohr radius $a^{*} \sim 100 \AA$ in GaAs. This potential shape holds valid whenever $N_{D}>5 \times 10^{10} \mathrm{~cm}^{-2}$, for which the exchange interaction on the subband population and energy is only very weak [16. Hence we can confidently neglect this effect hereafter.

In the effective-mass $\mathbf{k} \cdot \mathbf{p}$ approximation, the electronic wave function is written as a sum of products of band-edge orbitals with slowly varying envelope-functions. Keeping only the two nearby bands, there are two coupled envelope-functions describing the $s$-like conductionband and $p$-like valence-band states of the semiconductor, subject to an effective $2 \times 2$ Dirac Hamiltonian. Assuming that the band modulation depends only on $z$, the resulting equation for the envelope-functions in the conduction- and valence-bands can be written as follows 17,18

$$
\left[\begin{array}{cc}
E_{g} / 2-E+V(z) & -i \hbar v \partial \\
-i \hbar v \partial & -E_{g} / 2-E+V(z)
\end{array}\right]\left(\begin{array}{l}
f_{c}(z) \\
f_{v}(z)
\end{array}\right)=0
$$


where $\partial=d / d z, E_{g}=1.42 \mathrm{eV}$ is the gap of GaAs and $V(z)$ gives the gap center. Here the energy is measured from the gap center at $|z| \rightarrow \infty$. The velocity $v$ is related to the Kane's momentum matrix elements and is given by $v=\sqrt{E_{g} / 2 m^{*}}$. In particular, $\hbar v=9.0 \mathrm{eV} \AA$ in GaAs. It should be mentioned that the non-zero in-plane momentum can be easily absorbed in the parameter definitions and we will ignore it in what follows. It is worth mentioning that electrons and holes are treated within the same footing using this simple two-band model. This is so because the effects of other bands are not included in the Hamiltonian. However, it is known that significant spin-orbit coupling takes place in III-V semiconductor. Thus, a more elaborate treatment should include envelope-functions for three interactings bands — conduction, light hole and split-off hole- . Fortunately, a detailed treatment of a threeband semiconductor can be reduced to a two-band semiconductor by a suitable redefinition of the envelope-functions [19]. Hence a two-band model brings a simple way to study III-V semiconductors.

To solve Eq. (2) we use the ansatz [20]

$$
\left(\begin{array}{c}
f_{c}(z) \\
f_{v}(z)
\end{array}\right)=\left[\begin{array}{cc}
E_{g} / 2+E-V(z) & -i \hbar v \partial \\
-i \hbar v \partial & -E_{g} / 2+E-V(z)
\end{array}\right]\left(\begin{array}{c}
\phi(z) \\
\phi(z)
\end{array}\right),
$$

where the function $\phi(z)$ satisfies the equation

$$
\left\{-\hbar^{2} v^{2} \frac{d^{2}}{d z^{2}}+\frac{E_{g}^{2}}{4}-[E-V(z)]^{2}+i \hbar v \frac{d V(z)}{d z}\right\} \phi(z)=0,
$$

and the potential is given by (1). It is straightforward although tedious to demonstrate that the solutions can be expressed in terms of confluent hypergeometric functions $M(a, c ; x)$, as defined in Ref. [21]. However, for the sake of brevity we do not write down $\phi(z)$ explicitly and simply quote the final result. Once $\phi(z)$ is known, we can make use of Eq. (3) to obtain the envelope functions. Thus

$$
\left(\begin{array}{c}
f_{c}(z) \\
f_{v}(z)
\end{array}\right)=A_{+} \exp \left(-\frac{z q}{\alpha}+i \xi e^{-z / \alpha}\right)\left(\begin{array}{l}
G_{1}^{*}(-z)+\frac{i}{2} \frac{\epsilon_{g}}{q-i \epsilon} G_{0}^{*}(-z) \\
G_{1}^{*}(-z)-\frac{i}{2} \frac{\epsilon_{g}}{q-i \epsilon} G_{0}^{*}(-z)
\end{array}\right)
$$

for $z>0$ and 


$$
\left(\begin{array}{l}
f_{c}(z) \\
f_{v}(z)
\end{array}\right)=A_{-} \exp \left(\frac{z q}{\alpha}+i \xi e^{z / \alpha}\right)\left(\begin{array}{l}
G_{1}(z)-\frac{i}{2} \frac{\epsilon_{g}}{q+i \epsilon} G_{0}(z) \\
G_{1}(z)+\frac{i}{2} \frac{\epsilon_{g}}{q+i \epsilon} G_{0}(z)
\end{array}\right)
$$

for $z<0$, where $A_{ \pm}$are constants. For brevity we have defined the following dimensionless parameters $\epsilon=E \alpha / \hbar v, \epsilon_{g}=E_{g} \alpha / \hbar v, \xi=g \alpha / \hbar v$ and $q^{2}=\epsilon_{g}^{2} / 4-\epsilon^{2}$. The function $G_{k}(z)$ with $k=0,1$ is defined as follows

$$
G_{k}(z)=M\left(k+q+i \epsilon, 1+2 q, 2 i \xi e^{z / \alpha}\right) .
$$

The energies can be obtained by imposing the continuity of the envelope functions at $z=0$. In doing so, one finally gets

$$
E=\left(\frac{E_{g}}{2}\right) \cos \left(\lambda_{1}-\lambda_{0}\right)
$$

where $\lambda_{k}=\lambda_{k}(E)=\arg \left[G_{k}(0)\right]$, with $k=0,1$. Notice that $E$ is found by solving a transcendental equation using the usual search methods. In the range of parameters we have studied, a very fast convergence of the confluent hypergeometric series $M(a, b ; x)$ is attained, and thus only the first few terms must be computed. Therefore, solution of the transcendental equation (6) takes very short CPU times in most computers.

\section{RESULTS AND DISCUSSION}

As an illustration, we have calculated the binding energy of the ground subband $E_{b}$, defined as the difference in energy between the conduction-band edge far away from the $\delta$-layer and the ground electron subband, as a function of the donor concentration $N_{D}$. This binding energy is an important parameter since it can be readily determined experimentally, for instance, by deep level transient spectroscopy [22]. Results are shown in Fig. [1 in a log-log scale. As expected, the binding energy increases on increasing the doping level. Interestingly, the binding energy is of the form $E_{b}=11.184\left(N_{D} a^{* 2}\right)^{2 / 3} \mathrm{meV}$, that is, it scales as $N_{D}^{2 / 3}$, in a similar fashion that $g$ defined above. Analogous power law is found in V-shaped potential wells, often considered a good description of $\delta$-doped semiconductors, by means of ordinary 
one-band Hamiltonians within the envelope function approach [23]. However, Dirac-type Hamiltonians for V-shaped potential well cannot support quantum confined states because electron states can tunnel through to hole states, even for small electric fields [24, 25]. This phenomenon is similar to the well-known Klein paradox in quantum electrodynamics. Hence the use of (11) overcomes such difficulties while it retains most of the main features of subband energy in V-shaped potential (e.g., scaling law with $N_{D}$ ). Finally, let us comment the results when the same potential (11) is considered in a one-band framework, i.e., using the standard Ben Daniel-Duke Hamiltonian [26]. After some algebra, the binding energy of the ground subband $E_{b}$ can be obtained from the following transcendental equation involving Bessel functions $J_{\nu}(x)$

$$
J_{\nu+1}(\tilde{g})-J_{\nu-1}(\tilde{g})=0
$$

where $\nu=(2 \alpha / \hbar v) \sqrt{E_{b} E_{g}}$ and $\tilde{g}=(2 \alpha / \hbar v) \sqrt{g E_{g}}$. After finding numerically the roots of this equation, we obtain that in the one-band model the binding energy is given by $E_{b}=11.179\left(N a^{* 2}\right)^{2 / 3} \mathrm{meV}$. Two important points should be remarked. Firstly, once again the binding energy scales as $N_{D}^{2 / 3}$. Secondly, this binding energy is always smaller than that obtained in the two-band model for the same doping level. In other words, the subband in the one-band model is above the subband in the two-band model. The depression of levels when coupling of host bands is considered has also been found in sawtooth GaAs superlattices [8]. The explanation relies on the fact that the nonparabolicity effects imply an increment of the effective mass with energy and, consequently, the electronic levels are lowered. It is clear that the difference in energy we have found is not very large due to the wide gap of GaAs $\left(E_{g}=1.42 \mathrm{eV}\right)$ as compared to conduction-band modulation, which amounts up to $0.23 \mathrm{eV}$ for the higher doping level considered in the present study. Nevertheless, in other materials like Si:Sb, where the gap is narrower and high doping level can be reached, the difference should be much larger. 


\section{CONCLUDING REMARKS}

In conclusion, we have presented a theoretical study of $\delta$-doped semiconductors within the two-band model framework. As an example, we have considered Si $\delta$-doped GaAs, for which a number of theoretical and experimental results are available. However our present treatment should be valid in various $\delta$-doped semiconducting materials. A simple parametrization of the one-electron potential due to ionized dopant atoms allows us to find exact solutions for the envelope function and subband energy. One of the main conclusions

of the work is that the binding energy scales with $N_{D}^{2 / 3}$, similarly to which is found in solving the Schrödinger equation for the ideal V-shaped potential. We have also evaluated the effects of the coupling of host bands and obtained that they lead to a depression of the subband energy. This is a direct consequence of nonparabolicity effects, which manifest themselves via an increase of the effective mass with energy.

\section{ACKNOWLEDGMENTS}

This work is supported by CICYT (Spain) through project MAT95-0325. 


\section{REFERENCES}

[1] A. Zrenner, H. Reisinger, and K. Ploog, Proc. 17th Int. Conf. Physics of Semiconductors (San Francisco, 1984) edited by J. P. Chadi and W. A. Harrison, p. 325 (Springer, Berlin, 1985).

[2] T. E. Whall, Contemp. Phys. 33, 369 (1992).

[3] K. Ploog, J. Cryst. Growth. 81, 304 (1987).

[4] H. P. Zeindl, T. Wegehaupt, I. Eisele, H. Oppolzer, H. Reisinger, G. Tempel, and F. Koch, Appl. Phys. Lett. 50, 1164 (1987).

[5] W. Cheng, A. Zrenner, Q. Y. Ye, F. Koch, D. Grützmacher, and P. Balk, Semicond. Sci. Technol. 4, 16 (1989).

[6] J. Kortus and J. Monecke, Phys. Rev. B 49, 17216 (1994).

[7] G. Bastard, Phys. Rev. B 24, 5693 (1981).

[8] F. Domínguez-Adame and B. Méndez, Semicond. Sci. Technol. 9, 1358 (1994).

[9] L. Ioratti, Phys. Rev. B 41, 8340 (1990).

[10] J. C. Egues, J. C. Barbosa, A. C. Notari, P. Basmaji, L. Ioratti, E. Ranz, and J. C. Portal, J. Appl. Phys. 70, 3678 (1991).

[11] F. Domínguez-Adame and B. Méndez, Phys. Rev. B 49, 11471 (1994).

[12] M. H. Degani, Phys. Rev. B 44, 5580 (1991).

[13] A. Zrenner, F. Koch, and K. Ploog, Surf. Sci. 196, 671 (1988).

[14] J. A. Cuesta, A. Sánchez, and F. Domínguez-Adame, Semicond. Sci. Technol. 10, 1303 (1995).

[15] A. Gold, A. Ghazali, and J. Serre, Semicond. Sci. Technol. 7, 972 (1992). 
[16] P. M. Koenraad, F. A. P. Blom, C. J. G. M. Langerak, M. R. Leys, J. A. A. J. Perenboom, J. Singleton, S. J. R. M. Spermon, W. C. van der Vleuten, A. P. J. Voncken, and J. H. Wolker, Semicond. Sci. Technol. 5, 861 (1990).

[17] J. Callaway, Quantum Theory of the Solid State, (Academic Press, San Diego, CA) p. 33.

[18] R. Beresford, Semicond. Sci. Technol. 8, 1957 (1993).

[19] R. Beresford, Phys. Rev. B 49, 13663 (1994).

[20] R. P. Feynman and M. Gell-Mann, Phys. Rev. 109, 193 (1958).

[21] M. Abramowitz and I. Stegun, Handbook of Mathematical Functions, (Washington: US Government Printing Office, 1964).

[22] Q. S. Zhu, Z. T. Zhong, L. W. Lu, and C. F. Li, Appl. Phys. Lett. 65, 2425 (1994).

[23] E. F. Schubert, T. D. Harris, J. E. Cunningham, and W. Jan, Phys. Rev. B 39, 11011 (1989).

[24] A. Z. Capri and R. Ferrari, Can. J. Phys. 63, 1029 (1985).

[25] F. Domínguez-Adame and M. A. González, Europhys. Lett. 13, 193 (1992).

[26] G. Bastard, Wave Mechanics Applied to Semiconductor Heterostructures, (Les Editions de Physique, Les Ulis, France) p. 73. 


\section{FIGURES}

FIG. 1. Binding energy in Si $\delta$-doped GaAs as a function of the donor concentration in a two-band model. 